\title{
Effect of Ionic Liquid and Nanoparticles on PVA-co-PE-Based GPEs for the Applications in DSSCs
}

\author{
S. Ramesh ${ }^{(凶)}$, C. Y. Tan, and K. Ramesh \\ Centre for Ionics, Department of Physics, Faculty of Science, \\ University of Malaya, 50603 Kuala Lumpur, Malaysia \\ ramesh@um.edu.my
}

\begin{abstract}
Poly (vinyl alcohol-co-ethylene) (PVA-co-PE) was used as the host polymer together with $\mathrm{NaI}$ as the dopant salt. The maximum conductivity of $2.27 \pm 0.01 \mathrm{mS} \mathrm{cm}-1$ was achieved with $40 \mathrm{wt} \%$ of $\mathrm{NaI}$ salt. In respect to the 2nd system, the highest ionic conductivity $3.99 \pm 0.01 \mathrm{mS} \mathrm{cm}-1$ of was obtained by adding $10 \mathrm{wt} \%$ of MPII ionic liquid; meanwhile for the $3 \mathrm{rd}$ system, it exhibits the highest conductivity of $3.75 \pm 0.01 \mathrm{mS} \mathrm{cm}-1$ at $4 \mathrm{wt} \%$ of $\mathrm{Ni} 3$ (PO4)2 nanoparticles. According to temperature-dependence studies, the ionic conductivity of all samples is proportional to the temperature. Not only that, all samples did consistent with Arrhenius equation which indicates that the ion transport mechanism is hopping mechanism. Then, dielectric and modulus studies were carried out to further understand the electrical properties of the GPE and the results obtained were qualitatively similar among all the systems. Besides, FTIR spectroscopy studies have been carried out. Via the studies, the existence of complexation between PVA-co-PE, NaI, MPII, and Ni3(PO3)2 was confirmed. This is shown by the shifting of the characteristic peaks, changes in the intensity and existence of new peaks. For XRD studies, the results revealed the structural properties of the GPE samples. It was also observed that the samples with higher degree of crystallinity generally have a lower ionic conductivity owing to a lower mobility. The coherent length of each sample was calculated too. The results obtained from TGA show us that the addition of MPII ionic liquid and Ni3(PO4)2 increase the thermal stability of the GPE. Lastly, the synthesized GPEs were used to fabricate DSSC. For the iodide salt system, the highest efficiency of $3.32 \%$ was achieved with 40 wt $\%$ of NaI. By adding $10 \mathrm{wt} \%$ of MPII ionic liquid, the efficiency increases to $4.36 \%$. However, the best sample among three systems is N4. It has $4 \mathrm{wt} \%$ of Ni3(PO4)2 and an efficiency of $5.84 \%$, along with $j$ "\# of $14.21 \mathrm{~mA} \mathrm{~cm}-2, V \% \#$ of $0.66 \mathrm{~V}$ and fill factor of $62.5 \%$.
\end{abstract}

